\title{
EFICÁCIA DA REABILITAÇÃO VESTIBULAR EM IDOSOS COM TONTURA
}

\author{
Pâmela Camila PEREIRA ${ }^{1}$ \\ Luís Henrique Sales OLIVEIRA ${ }^{2}$ \\ Valdomiro Vagner de SOUZA ${ }^{3}$ \\ Alexandre de Souza e SILVA ${ }^{4}$
}

1 Fisioterapeuta - UNIVÁS, Especialista em Fisioterapia Pneumofuncional -UGF, Pós-Graduanda em Fisioterapia Neurofuncional - FEPI, Aluna Especial do Programa de Pós-Graduação em Reabilitação do Equilíbrio Corporal e Inclusão Social - UNIBAN, pam_milaf@yahoo.com.br.

${ }^{2}$ Fisioterapeuta - UNIP, Doutor em Ciências - UNIFESP, docente dos Cursos de Graduação e Pós-Graduação - FEPI, lhfisio@ig.com.br

${ }^{3}$ Farmacêutico - UNIVÁS, Mestre em Cîências Farmacêuticas - UNIFAL, docente de Graduação e Pós-Graduação - FEPI, valdomirovagner@gmail.com

${ }^{4}$ Profissional de Educação Física - UNITAU, Mestre em Ciências Biológicas - UNIVAP, docente de Graduação e PósGraduação da FEPI, alexprofmestre@yahoo.com.br

Recebido em: 30/10/2013 - Aprovado em: 21/12/2013 - Disponibilizado em: 15/01/2014

RESUMO: A reabilitação vestibular (RV) visa diminuir a tontura e a instabilidade corporal e tem se mostrado importante e efetiva estratégia no tratamento do paciente com desordens do equilíbrio corporal. Verificar os benefícios dos exercícios de RV por meio de uma revisão de literatura. Foi realizada pesquisa nas bases de dados Medline, IBECS, Cochrane, Lilacs e Scielo. Os artigos foram escolhidos através de seus conteúdos, devendo estar relacionados ao tema proposto neste estudo e nível de evidência A e/ou B nacional e internacional. Nossos achados corroboram a literatura ao demonstrar a efetividade dos programas de RV quanto à melhoria da qualidade em idosos com tonturas, tornando uma importante alternativa não só terapêutica como também de promoção de saúde para os indivíduos que sofram desse agravo. Com base no estudo apresentado observou-se grande sucesso da RV no idoso com tontura, causando um efeito psicológico positivo com a recuperação da segurança física e psíquica e consequente melhora da qualidade de vida.

Palavras-chave: Idoso. Reabilitação. Equilíbrio Postural. Tontura. Fisioterapia.

\begin{abstract}
Vestibular rehabilitation (VR) aims at reducing dizziness and instability body and has been an important and effective strategy in the treatment of patients with disorders of body balance. To assess the benefits of VR exercises through a literature review. A survey was conducted in Medline, IBECS, Cochrane, Lilacs and SciELO. The articles were chosen by their content, and should be related to the theme proposed in this study and evidence level A and / or B and abroad. Our findings corroborate the literature to demonstrate the effectiveness of VR programs in improving the quality in elderly patients with dizziness, becoming an important alternative not only therapeutic but also health promotion for individuals who suffer from this condition. Based on the study presented observed great success of RV in the elderly with dizziness, causing a positive psychological effect with the recovery of physical and psychological security and consequent improvement in quality of life.
\end{abstract}

Keywords: Aging. Rehabilitation. Postural Balance. Dizziness. Physiotherapy.

\section{INTRODUÇÃO}

A população de idosos representa um contingente de quase 15 milhões de pessoas com 60 anos ou mais de idade $(8,6 \%$ da população brasileira). Nos próximos 20 anos, a população idosa do Brasil poderá ultrapassar os 30 milhões de pessoas e deverá representar 
quase $13 \%$ da população ao final deste período (IBGE, 2013).

$\mathrm{O}$ envelhecimento pode comprometer a habilidade do sistema nervoso central em processar os sinais vestibulares, visuais e proprioceptivos responsáveis pela manutenção do equilíbrio corporal, diminuindo a capacidade de modificações dos reflexos adaptativos. Tal processo degenerativo é responsável pela ocorrência de vertigem e/ou tontura e de desequilíbrio na população geriátrica, sendo este um dos principais fatores que limitam a vida do indivíduo idoso (RUWER, ROSSI, SIMON; 2005).

A tontura é a sensação de perturbação do equilíbrio corporal, enquanto a vertigem é uma sensação de desorientação espacial do tipo rotatória. A vertigem e outras tonturas de origem vestibular estão presentes em 5 a $10 \%$ da população mundial, sendo o sintoma mais comum após os 65 anos, atingindo $80 \%$ dos idosos atendidos em ambulatórios geriátricos. A literatura estima que a prevalência da tontura na população com mais de 65 anos chegue a $85 \%$, sendo ainda que as fraturas decorrentes de quedas nesta população são responsáveis por $70 \%$ das mortes que ocorrem acidentalmente (GAZZOLA et al., 2005; MANTELLO et al., 2008; MELO et al., 2013).

No idoso a tontura é considerada uma síndrome geriátrica, condição de saúde multifatorial, decorrente de alterações próprias do envelhecimento e/ou patológicas, a qual resulta em instabilidade e maior predisposição às quedas. Entretanto, já aos 40 anos, é possível observar alterações microscópicas sinápticas no nervo vestibular; aos 50 anos há o aumento da degeneração dos receptores vestibulares nas cristas dos canais semicirculares e sáculo e aos 60 anos, dentre tantas alterações, pode-se citar o aumento do atrito das fibras nervosas do nervo vestibular e a redução da velocidade de condução do estímulo elétrico no nervo vestibular (GAZZOLA et al., 2006).

Um dos principais fatores que limitam a vida do indivíduo idoso é o desequilíbrio corporal, que em aproximadamente $80 \%$ dos casos não pode ser atribuído a uma causa específica, mas ao comprometimento do sistema de equilíbrio como um todo (MIRALLAS et al., 2011).

O tratamento dos idosos que apresentam limitações de locomoção minimiza o número de acidentes como quedas, favorecendo a sensação de independência e de orientação espacial. Para as vestibulopatias, existem opções terapêuticas como o uso de medicação antivertiginosa, tratamento cirúrgico, orientação nutricional, mudança de hábitos inadequados, psicoterapia e reabilitação vestibular (RV) (MIRALLAS et al., 2011; ZANARDINI et al., 2007).

A RV tem sido evidenciada por agir fisiologicamente sobre o sistema vestibular, sendo um recurso terapêutico pela sua proposta 
de atuação baseada em mecanismos centrais de neuroplasticidade conhecidos como adaptação, habituação e substituição para obtenção da compensação vestibular. Visa diminuir a tontura e a instabilidade corporal e tem se mostrado importante e efetiva estratégia no tratamento do paciente com desordens do equilíbrio corporal, melhorando a competência e o bem estar na realização das atividades de vida diárias (AVD's) e proporcionando uma acentuada melhora na qualidade de vida (CUNHA, SETTANNI, GANANCA, 2005; PATATAS, GANANÇA, GANANÇA, 2009).

Os principais objetivos da RV são: promover a estabilização visual e aumentar a interação vestíbulo visual durante a movimentação da cabeça; proporcionar uma melhor estabilidade estática e dinâmica nas situações de conflito sensorial e diminuir a sensibilidade individual durante a movimentação cefálica (GANANÇA et al., 2004; REZENDE et al., 2003).

A RV pode promover a cura completa em $30 \%$ dos casos e diferentes graus de melhora em 85\% dos indivíduos (GANANÇA et al., 2004). É importante ressaltar que esse problema atinge um número significativo de idosos, que vem aumentando gradativamente em todo o mundo. Sendo a tontura um dos sintomas sensoriais de maior incidência nos idosos, o objetivo desta pesquisa foi verificar os benefícios dos exercícios de RV por meio de uma revisão de literatura.

\section{MATERIAIS E MÉTODOS}

Foi realizada pesquisa nas bases de dados Medline, IBECS, Cochrane, Lilacs e Scielo. Os artigos foram escolhidos através de seus conteúdos, devendo estar relacionados ao tema proposto neste estudo e nível de evidência A e/ou B nacional e internacional, utilizando os descritores: Idoso, Reabilitação, Equilíbrio Postural, Tontura, e Fisioterapia. Trata-se de um estudo de revisão de literatura, onde foram analisados os mais relevantes estudos publicados originalmente na língua inglesa e portuguesa, durante o século XX (2001 a 2013).

Para identificar os delineamentos dos estudos, foram empregados os seguintes termos: randomized controlled trial, review e meta-analysis.

\section{RESULTADOS}

\section{ENVELHECIMENTO}

O envelhecimento pode ser considerado como um processo dinâmico e progressivo, no qual ocorrem alterações morfológicas, funcionais, bioquímicas que alteram 
progressivamente o organismo, tornando o mais suscetível às agressões intrínsecas e extrínsecas que terminam por levá-lo à morte (MANTELLO et al., 2008).

Os pacientes idosos são os mais acometidos pelas doenças do labirinto levando a incapacidades funcionais. Segundo Soares (2007), 40\% das pessoas acima dos 40 anos eram acometidas por doenças do labirinto. $61 \%$ eram de mulheres idosas com mais de 70 anos de idade apresentando vertigem, sendo que 50 a $60 \%$ delas viviam em seus domicílios.

A literatura estima que a prevalência da tontura na população com mais de 65 anos chegue a $85 \%$, sendo ainda que as fraturas decorrentes de quedas nesta população são responsáveis por $70 \%$ das mortes que ocorrem acidentalmente (MANTELLO et al., 2008).

$\mathrm{O}$ número de idosos do sexo feminino tem aumentado em relação ao número de idosos do sexo masculino, o que permite a essas mulheres ficarem mais expostas a riscos de quedas devido à execução das atividades ocupacionais do lar. Este fenômeno pode ser explicado porque durante a realização das atividades, ocorre a execução de movimentos cefálicos que associam a extensão com a rotação e inclinação da cabeça, promovendo o aparecimento das tonturas (SOARES, 2007).

Com relação à tontura, estima-se que uma em cada dez pessoas no mundo tem ou teve tontura de origem vestibular. A tontura constitui o segundo sintoma de maior prevalência, perdendo em frequência para a cefaleia; dos 65 anos em diante, é o sintoma de maior prevalência. Em pessoas com mais de 75 anos, a prevalência é da ordem de $80 \%$ (GANANÇA; CAOVILLA; GANANÇA, 2002).

Várias alterações contribuem para as tão frequentes queixas de tonturas nos idosos, como redução da mobilidade da coluna vertebral, contraturas na região cervical, redução do fluxo sanguíneo arterial, redução da capacidade proprioceptiva, degeneração auditiva, vestibular e visual, dificuldade de alimentação, transtornos depressivos, entre outros quadros que afetam direta ou indiretamente o equilíbrio do paciente (MANTELLO et al., 2008; MITRE, 2003).

\section{REABILITAÇÃO VESTIBULAR}

Estudos tem mostrado que a intervenção terapêutica, por meio de exercícios de reabilitação, pode ser favoravelmente adotada, frente às queixas de alterações do equilíbrio corporal de idosos portadores de disfunções vestibulares periféricas de causa idiopática, aumentando a qualidade de vida dos pacientes (MANTELLO et al., 2008; MIRALLAS et al., 2011). 
A RV vem sendo uma das técnicas utilizadas com melhores resultados apresentados para resolução das alterações labirínticas. Pretende restaurar a função de equilíbrio ou trazê-la o mais próximo possível do normal, sem a terapia medicamentosa. Com objetivos significativos na melhora do equilíbrio global, qualidade de vida e a restauração da orientação espacial por meio de exercícios que estimulam os fenômenos de adaptação envolvendo a habituação, substituição, adaptação e a compensação, implementando novos arranjos nas informações sensoriais periféricas, permitindo novos padrões de estimulação vestibular necessários para realização das atividades de forma automática e precisa (MIRALLAS et al., 2011; SOARES, 2011).

Segundo Mirallas e seus colaboradores (2011), o treinamento dessas funções torna mais eficiente às reações de equilíbrio. Diminuem os desequilíbrios posturais e, consequentemente, as quedas, já que utiliza movimentos cefálicos, cervicais e oculares, nas posturas sentada, em apoio bipodal, unipodal e durante a deambulação, em superfícies instáveis, com diminuição da sensação proprioceptiva dos pés, dos olhos, além de posturas provocativas da vertigem e da tontura.

A meta dos protocolos de RV inclui a melhora do equilíbrio funcional e da mobilidade, da condição física geral e da aptidão total, realização do cuidado e deambulação independente, melhora das habilidades para execução das AVD's e diminuição dos sintomas associados às desordens do ouvido interno. Com o surgimento da terapia de RV diminuíram essas incapacidades funcionais e suas consequências (SOARES, 2011).

\section{DISCUSSÃO}

Estudos randomizados com intervenção por RV específica para a população idosa com tonturas são restritos na literatura. Entretanto, apesar da escassez em números, os artigos selecionados nesta revisão mostraram evidências positivas da RV no controle postural, capacidade funcional e qualidade de vida de idosos com queixas ou diagnóstico de síndrome vestibular. Entretanto, a diferença metodológica entre eles torna difícil estabelecer o melhor protocolo, tempo de intervenção, entre outros parâmetros.

As propostas de intervenção utilizadas pela maioria dos estudos foram o protocolo de Cawthorne \& Cooksey, exercícios de adaptação da Herdman e exercícios de equilíbrio corporal estático e dinâmico. Essas propostas têm como objetivo promover a estabilização visual aos movimentos da cabeça, melhorar a estabilidade postural nas situações em que surgem os conflitos sensoriais, minimizar a sensibilidade à 
movimentação cefálica e melhorar o equilíbrio corporal estático e dinâmico. Entre os estudos que utilizaram como intervenção o protocolo de Cawthorne \& Cooksey, houve melhora significativa em relação ao grupo controle no equilíbrio dinâmico, na posturografia e na escala de vida diária (HERDEMAN, 2012; SIMOCELI, BITTAR, SZNIFER, 2008; HERDMAN, HALL, SCHUBERT, 2007).

Ganança, Dias e Ganança (2001), observou-se que investir tempo num adequado aconselhamento ao paciente vertiginoso é um fator imprescindível para adesão ao tratamento por parte dos pacientes, pois esta etapa é tão importante quanto à realização dos exercícios de RV, para que o paciente acredite no tratamento, realize os exercícios, acelere a compensação e mantenha os bons resultados obtidos após a alta.

A eficácia de RV nos idosos vão de acordo com Herdeman, Hall e Schubert (2007), Zanardini et al., (2007) e Gazzola et al., (2006). Assim como Ganança et al., (2004) e Sznifer et al., (2004) observou-se incrementos em todos os escores avaliados pelo DHI (Dizziness Handicap Inventory ) após a RV em idosos, detectando mudança na qualidade de vida dos mesmos. Diante destes resultados, não se considera que exista limite de idade para submeter pacientes vertiginosos ao programa de RV.
Evidencia-se a importância de se avaliar os prejuízos da qualidade de vida em pacientes com tonturas, especialmente idosos, com objetivo de quantificar os efeitos impostos nas funções de vida do dia a dia, além de auxiliar na escolha do tratamento e avaliação do mesmo.

Conforme já descrito por Lobos (2002), em um estudo sobre a RV em grupo de idosas com idade igual ou superior a 60 anos, a RV é de baixo custo, mas foi efetiva em produzir modificações no equilíbrio e na habilidade funcional das idosas, podendo ser aplicada na promoção da saúde e na melhora da qualidade de vida dos idosos.

Rezende et al., (2003) e Ganança et al., (2004) evidenciaram que a RV não desencadeia efeitos colaterais e mostrou ser um caminho terapêutico extremamente importante com melhora do convívio e da integração social. (GANANÇA et al., 2004).

Cohen et al., (2000), Simoceli, Bittar, Sznifer (2008) referem a escassez de estudos relacionando a qualidade de vida dos indivíduos com desordens vestibulares. O tratamento por meio da RV é cada vez mais enfatizado pela literatura internacional, porque além de melhorar o equilíbrio do idoso com tonturas, tem função profilática. No Brasil, nos últimos anos, a RV tem sido muito utilizada e seus protocolos têm sido diversificados e 
personalizados às necessidades dos pacientes portadores de vestibulopatias.

Nossos achados corroboram a literatura ao demonstrar a efetividade dos programas de RV quanto à melhoria da qualidade em idosos com tonturas, tornando uma importante alternativa não só terapêutica como também de promoção de saúde para os indivíduos que sofram desse agravo.

\section{CONCLUSÃO}

Com base nos artigos estudados observou-se grande sucesso da RV no idoso com tontura, causando um efeito psicológico positivo com a recuperação da segurança física e psíquica e consequente melhora da qualidade de vida.

Assim, levando em consideração os dados epidemiológicos do envelhecimento no Brasil e sabendo que grande parte dos idosos tem afecções otoneurológicas, conclui-se que a RV pode ser utilizada de modo benéfico nesta população e por isso justifica-se a importância do tratamento fisiológico com a participação dos diferentes profissionais da saúde que atuam na equipe gerontológica.

\section{REFERÊNCIAS}

BRASIL. Instituto Brasileiro de Geografia e Estatística. Perfil dos idosos responsáveis pelos domicílios no Brasil. Brasília: IBGE, 2005. Disponível em:

http://www.ibge.gov.br/home/estatistica/popula cao/projecao_da_populacao/default.shtm>.

Acesso em: 03 de setembro de 2013.

CUNHA F, SETTANNI FAP, GANANCA FF. What is the effect of Dizziness on the quality of life for patients whit Meniere`s disease? Rev Laryngol Otol Rhinol. 2005;126(3):155-8.

GANANÇA CF, DIAS CFG, GANANÇA MM. Orientação nutricional e mudanças de hábitos do paciente vertiginoso. In: Ganança MM. Estratégias terapêuticas em otoneurologia. São Paulo: Atheneu; 2001:55-66.

GANANÇA MM, CAOVILLA HH, GANANÇA CF. Vertigem e sintomas correlacionados, avaliação funcional do sistema vestibular. In: Ganança MM, coordenador. Vertigem: abordagens diagnósticas e terapêuticas. São Paulo: Lemos; 2002.

GANANÇA, F.F.; CASTRO, A. S. O.; BRANCO, F. C.; NATOUR, J. Interferência da tontura na qualidade de vida de pacientes com síndrome vestibular periférica. $R$. Bras.

Otorrinolaringol., São Paulo, v. 70, n. 1, p. 94101, fev, 2004.

GAZZOLA JM, GANANÇA FF, PERRACINI MR, ARATANI MC, DORIGUETO RS, GOMES CMC. O envelhecimento e o sistema vestibular. Fisioter Mov. 2005;18(5):39-48.

GAZZOLA JM, PERRACINI MR, GANANÇA MM, GANANÇA FF. Fatores associados ao equilíbrio funcional em idosos com disfunção vestibular crônica. Rev Bras Otorrinolaringol. 2006;72(3):983-90.

HERDMAN SJ. Reabilitação vestibular. São Paulo: Manole; 2002.

HERDMAN SJ, HALL CD, SCHUBERT MC, DAS VE, TUSA RJ. Recovery of dynamic 
visual acuity in bilateral vestibular

hypofunction. Arch Otolaryngol Head Neck

Surg. 2007;133(4):383-9.

LOBOS MB. Uma proposta de reabilitação

vestibular em grupo para idosas

institucionalizadas. Santa Maria (RS):

Universidade Federal de Santa Maria; 2002.

MANTELLO EB, MORIGUTI JC, RODRIGUES-JÚNIOR AL, FERRIOLI E.

Efeito da reabilitação vestibular sobre a qualidade de vida de idosos labirintopatas. Rev

Bras Otorrinolaringol 2008;74(2):172-80.

MELO NETO JS; STROPPA AEZ; PARRERA

CA; MAXIMIANO WF; HIDALGO CA.

Reabilitação Vestibular em portadores de

Vertigem Posicional Paroxística Benigna. Rev.

CEFAC v.15 n.3 São Paulo May/June 2013.

MIRALLAS NDR; CONTI MHS; VITTA A;

LAURENTI R; SAES SO. Avaliação e reabilitação vestibular no indivíduo idoso. Rev. Bras. Geriatr. Gerontol., rio de janeiro, 2011; 14(4):687-698.

MITRE EI. Aspectos Otorrinolaringológicos do idoso. In: Susuki HS. Conhecimentos essenciais para atender bem o paciente idoso. São Paulo:

Pulso Editorial; 2003. p. 25-32. 